\title{
c-met is overexpressed in type I ovarian cancer: Results of an investigative analysis in a cohort of consecutive ovarian cancer patients
}

\author{
MARCO JOHANNES BATTISTA ${ }^{1}$, MARCUS SCHMIDT ${ }^{1}$, SINA JAKOBI ${ }^{1}$, CRISTINA COTARELO ${ }^{2}$, \\ KATRIN ALMSTEDT ${ }^{1}$, ANNE-SOPHIE HEIMES ${ }^{1}$, GEORGIOS-MARIOS MAKRIS ${ }^{1}$, VERONIKA WEYER ${ }^{3}$, \\ ANTJE LEBRECHT $^{1}$, GERALD HOFFMANN ${ }^{1}$ and MICHAEL EICHBAUM ${ }^{4}$
}

\begin{abstract}
Departments of ${ }^{1}$ Gynecology and Obstetrics, and ${ }^{2}$ Pathology; ${ }^{3}$ Institute of Medical Biostatistics, Epidemiology and Informatics, University Medical Center Mainz, Mainz D-55131; ${ }^{4}$ Department of Gynecology and Obstetrics,

Marienkrankenhaus Frankfurt, Frankfurt D-60318, Germany
\end{abstract}

Received July 9, 2015; Accepted April 22, 2016

DOI: $10.3892 / 01.2016 .4895$

\begin{abstract}
The tyrosine kinase c-met alters signaling cascades such as the BRAF-MAPK and PI3K-PKB pathways. These alterations are involved in the carcinogenesis of type I but not type II ovarian cancer (OC). Therefore, the present study investigated the patterns of c-met expression in a cohort of consecutive patients with OC. c-met expression was determined by immunohistochemical analysis. Differences in c-met overexpression among subgroups of established clinicopathological features, including age, histological subtype, tumor stage, histological grading, post-operative tumor burden and completeness of chemotherapy, were determined by $\chi^{2}$ test. Cox regression analyses were performed to determine the prognostic effect of c-met. Survival rates were estimated using the Kaplan-Meier method. A total of 106 patients were enrolled into the study. c-met was overexpressed in $20.8 \%$ of the entire cohort; $35.7 \%$ of patients with type I OC and $8.6 \%$ of patients with type II OC showed overexpression $(\mathrm{P}=0.001)$. However, c-met overexpression was not associated with any other established clinicopathological features (all P-values $>0.05$ ). Univariate Cox regression analysis showed that overexpression of c-met was associated neither with progression-free survival (PFS) nor with disease-specific survival (DSS) $(\mathrm{P}=0.835$ and $\mathrm{P}=0.414$, respectively). Kaplan-Meier plots also failed to demonstrate an effect of c-met on the 5-year PFS and DSS rates $(\mathrm{P}=0.938$ and $\mathrm{P}=0.412$, respectively). These findings support the hypotheses that the overexpression of c-met is
\end{abstract}

Correspondence to: Dr Marco Johannes Battista, Department of Gynecology and Obstetrics, University Medical Center Mainz, Langenbeckstraße 1, Mainz D-55131, Germany

E-mail: battist@uni-mainz.de

Key words: ovarian cancer, c-met, type I ovarian cancer, type II ovarian cancer, prognosis associated with type I but not type II OC, and that overexpression of c-met does not affect the prognosis of OC.

\section{Introduction}

Ovarian cancer (OC) has the worst prognosis among the gynecological malignancies (1). Each year, 21,980 new cases are diagnosed in the United States and $\sim 14,270$ women succumb to OC (1). During the last decade, novel insights have led to a dualistic model of the carcinogenesis of OC (2-5). This model assists in the grouping of various different histological subtypes into two broad categories $(3,4)$. Low-grade serous $\mathrm{OC}$, endometrioid OC, clear cell OC and mucinous OC represent type I OC (3-5). These conditions generally present as a large tumor that is confined to a single ovary. Mutations in isoform b of rapidly accelerated fibrosarcoma protein (BRAF), phosphatidylinositol-4,5-bisphosphate 3-kinase catalytic subunit $\alpha$ (PI3KCA), catenin $\beta-1$ (CTNNB1), phosphatase and tensin homolog, Kirsten rat sarcoma viral oncogene homolog, and AT-rich interactive domain-containing protein $1 \mathrm{~A}$ are responsible for a step-wise progression from normal epithelium through differing degrees of atypia to non-invasive and then invasive type I carcinoma (3-5). Type II OC mainly consists of high-grade serous OC and presents as advanced disease with a poor prognosis (3-5). In the majority of type II OCs, alterations in p53 are responsible for genetic instability, with marked chromosomal aberrations (6).

Hepatocyte growth factor/scatter factor (HGF/SF) and its receptor tyrosine kinase, c-met, the product of the c-met proto-oncogene, provide vital signals for survival and long-distance epithelial and myogenic precursor cell migration during embryogenesis (7). Cancer cells are able to use HGF/SF-c-met for invasion and metastasis (7). Aberrant c-met activation results from various mechanisms and occurs in a variety of different types of cancer, including renal cancer, hepatocellular cancer, basal-like breast cancer, lung cancer and OC (8). The intracellular signaling cascades activated by c-met include the phosphoinositide 3-kinase-protein kinase B 
(PI3K-PKB) and the RAS-mitogen-activated protein kinase (MAPK) pathways $(7,9)$. A complex cross-signaling network involving the c-met epidermal growth factor receptor, c-met-vascular endothelial growth factor receptor (VEGFR) and c-met-Wingless-related integration site-CTNBB1 pathways has also emerged $(10,11)$. According to the results of a phase II trial, cabozantinib, a potent inhibitor of c-met and VEGFR-2, exhibited clinical activity in $68 \mathrm{OC}$ patients, as suggested by the recorded response rates (12). However, the prognostic impact of c-met in OC remains controversial (13-15).

Therefore, the present study examined the expression of c-met and its prognostic effect in an unselected, consecutive cohort of patients with $\mathrm{OC}$.

\section{Materials and methods}

Patients and tissue samples. The archives were searched for all patients with OC who underwent primary surgery at University Medical Center Mainz between 2004 and 2011. Patients entered the study only if formalin-fixed, paraffin-embedded (FFPE) tissue samples were available. Follow-up was performed by writing letters to patients or their physicians and by checking the patient records until May 2013. Mortality from OC or other reasons unrelated to cancer, and recurrence of disease, which included metastasis and local relapse, was documented. Patient charts were reviewed to collect data regarding age at diagnosis, histological grade, tumor stage of disease in accordance to the International Federation of Gynecology and Obstetrics guidelines, as previously reported $(16,17)$. Briefly, the amount of residual disease subsequent to primary surgery was registered as no tumor burden (R0), or as residual disease $<2 \mathrm{~cm}$ (R1) or $>2 \mathrm{~cm}$ (R2). Completed chemotherapy was defined as 6 courses of platinum-based monotherapy in early OC or as platinum-based combination therapy with paclitaxel for patients with advanced OC. Pathological review of all cases was performed by one individual in order to determine the histological type: In accordance with the current FIGO classification from 2014, all serous OC were classified as high-grade or low-grade serous OC as suggested by Malpica et al (18). In contrast, the current FIGO classification relies on the traditional three-tier grading system from 1971 for the mucinous, endometrioid, clear-cell and mixed subtypes (3-5). The study was approved by the Research Ethics Committee of the University Medical Centre Mainz (Mainz, Germany). Informed consent was obtained from all patients. All specimens were handled according to the ethical and legal standards.

Immunohistochemistry and evaluation of c-met staining. Immunohistochemical analyses were performed on $4-\mu \mathrm{m}$ thick sections according to standard procedures. Serial sections of FFPE tumor tissues were stained with polyclonal anti-HGF R/c-met goat anti-human antibodies (catalog no. AF276; R\&D Systems GmbH, Wiesbaden, Germany). The immunoreaction was visualized using Histofine ${ }^{\circledR}$ Simple Stain MAX peroxidase anti-goat antibody (catalog no. 414162F; Medac, Tornesch, Germany). Immunohistochemistry assays were performed in accordance with the

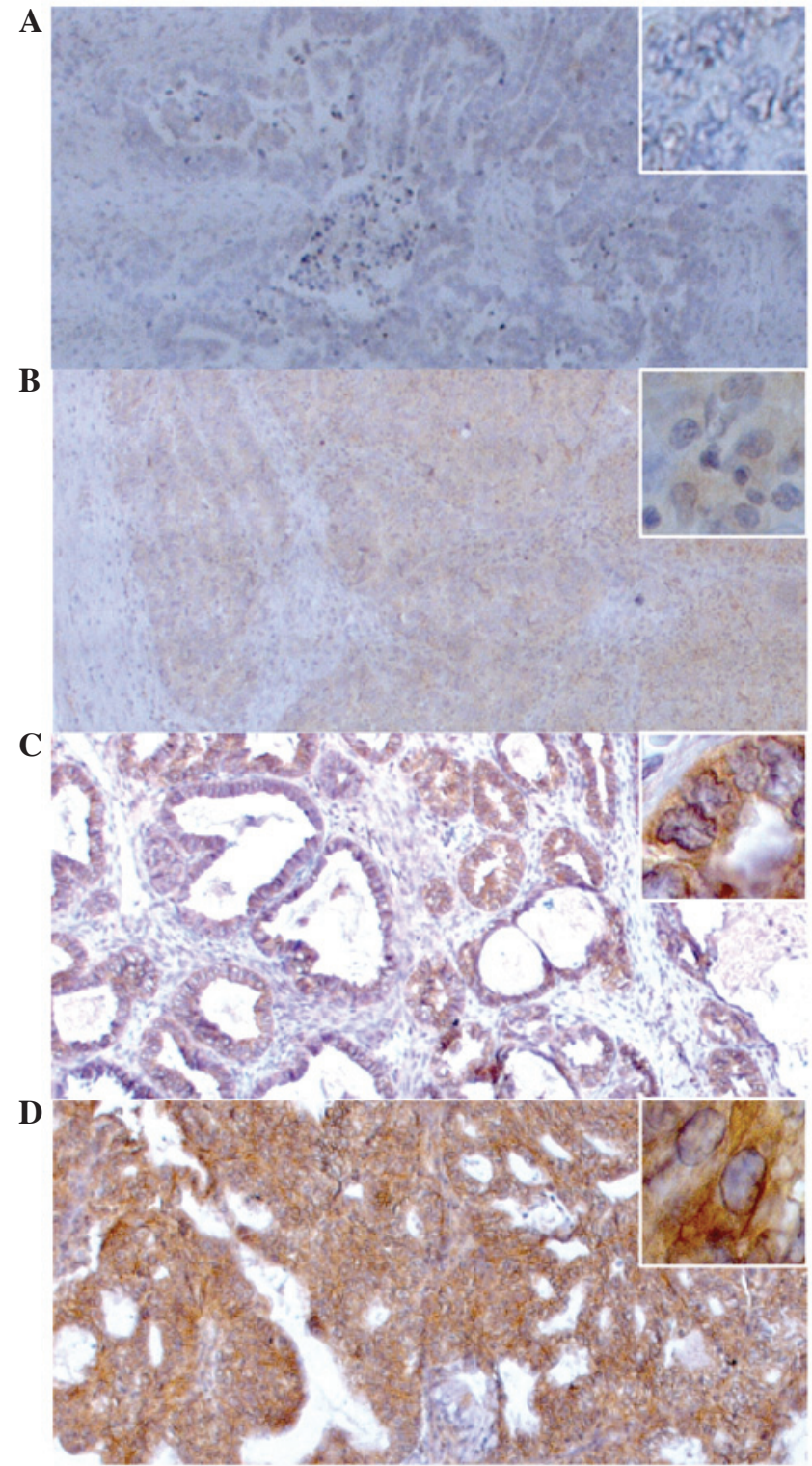

Figure 1. Representative examples of immunostaining (original magnification, x50; inset, x400). (A) No staining against c-met in a case of high-grade serous OC, histological grade 3. (B) Weak staining of c-met in a case of high-grade serous OC, histological grade 3. (C) Moderate staining of c-met in a case of low-grade serous OC, histological grade 1. (D) Strong staining of c-met in a case of low-grade serous OC. OC, ovarian cancer.

manufacturer's protocols. All series included appropriate positive (human liver tissues) and negative (distilled water used as a substitute for anti-HGF-R antibodies in human liver tissues) controls with adequate results.

c-met was assessed using a four-tier system, as previously described by Yamamoto et al for c-met in OC (13). Briefly, no discernible staining or background type staining was considered as negative (score of 0 ); definite cytoplasmic staining and/or equivocal discontinuous membrane staining scored 1+; unequivocal membrane staining with mild to moderate intensity scored 2+; and strong and complete membrane staining scored 3+ (Fig. 1). c-met was assessed as $2+$ or $3+$ only if at least $10 \%$ of the tumor cells showed complete membrane staining (13). A score of $2+$ or $3+$ was defined as c-met overexpression in accordance to the criteria 
Table I. Patient characteristics $(n=106)$.

\begin{tabular}{|c|c|}
\hline Parameter & Value \\
\hline \multicolumn{2}{|l|}{ Age, years } \\
\hline Mean $( \pm \mathrm{SD})$ & $59.08( \pm 12.60)$ \\
\hline Interquartile range & $51.44-70.36$ \\
\hline \multicolumn{2}{|c|}{ Tumor stage (FIGO), n (\%) } \\
\hline I & $22(20.8)$ \\
\hline II & $6(5.7)$ \\
\hline III & $63(59.4)$ \\
\hline IV & $15(14.2)$ \\
\hline \multicolumn{2}{|c|}{ Histological grade, $\mathrm{n}(\%)^{\mathrm{a}}$} \\
\hline G1 & $3(12.5)$ \\
\hline $\mathrm{G} 2$ & $11(45.8)$ \\
\hline G3 & $10(41.7)$ \\
\hline \multicolumn{2}{|l|}{ Histological type, n (\%) } \\
\hline Type I & $48(45.3)$ \\
\hline Type II & $58(54.7)$ \\
\hline Serous & $82(77.4)$ \\
\hline High-grade serous & $58(54.7)$ \\
\hline Low-grade serous & $24(22.6)$ \\
\hline Mucinous & $14(13.2)$ \\
\hline Endometrioid & $5(4.7)$ \\
\hline Clear cell & $2(1.9)$ \\
\hline Mixed & $3(2.8)$ \\
\hline \multicolumn{2}{|c|}{ Post-operative residual tumor burden, n (\%) } \\
\hline R0 & $62(58.5)$ \\
\hline $\mathrm{R} 1$ & $33(31.1)$ \\
\hline $\mathrm{R} 2$ & $11(10.4)$ \\
\hline \multicolumn{2}{|l|}{ Chemotherapy, n (\%) } \\
\hline Complete & $82(77.4)$ \\
\hline Incomplete & $13(12.3)$ \\
\hline Missing data & $11(10.4)$ \\
\hline \multicolumn{2}{|l|}{ Events, n (\%) } \\
\hline Relapse & $69(65.1)$ \\
\hline Mortality due to OC & $44(41.5)$ \\
\hline c-met-positive & $22(20.8)$ \\
\hline
\end{tabular}

${ }^{\mathrm{a}} \mathrm{n}=24$, the histological grading is given only for mucinous, endometrioid, clear-cell and mixed cases in accordance to the current FIGO classification. SD, standard deviation; FIGO, International Federation of Gynecology and Obstetrics; R0, no tumor burden; R1, residual disease $<2 \mathrm{~cm}$; R2, residual disease $>2 \mathrm{~cm}$; OC, ovarian cancer.

reported by Yamamoto et al (13). The immunohistochemical evaluation was performed independently by two individuals trained in histological and immunohistochemical diagnostics, who were unaware of the clinical outcome. Slides with a different assessment were discussed until a consensus was reached.

Statistical analysis. Statistical analyses were performed using the SPSS statistical software program, version 23.0 (IMB SPSS, Armonk, NY, USA). Patient characteristics are

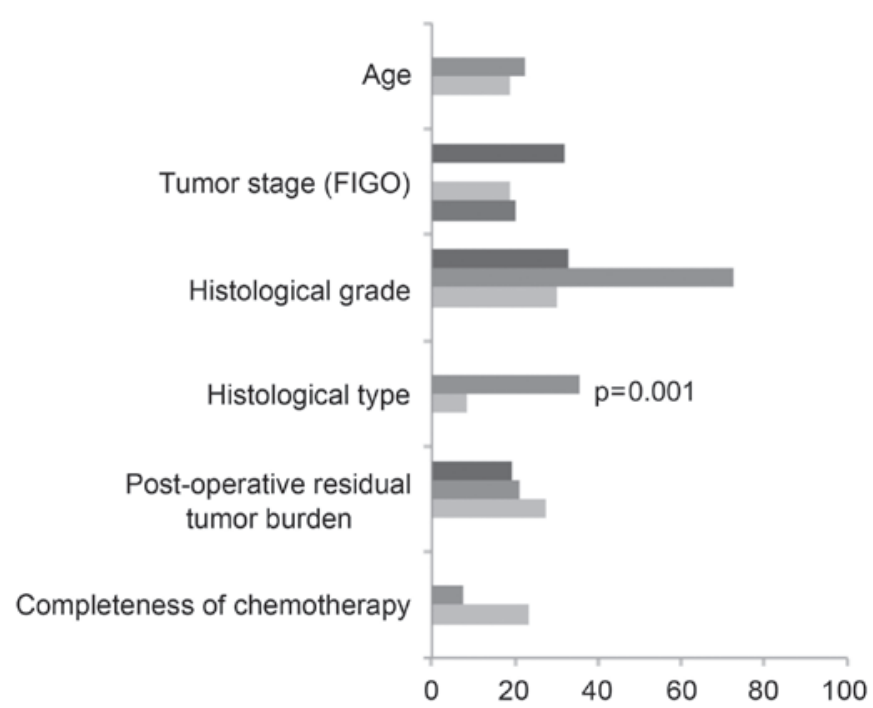

Figure 2. Overexpression of c-met with regard to clinicopathological factors. Age is categorized by the mean; tumor stage is classified in accordance to FIGO criteria and is categorized into stage I, II, III and IV; histological grade is categorized into grade I, II and III and is provided only for non-serous OC $(n=24)$; histological type is categorized into type I and type II OC; post-operative residual tumor burden is categorized into R0 (defined as no post-operative tumor burden), R1 (defined as tumor burden $<2 \mathrm{~cm}$ ) and R2 (defined as tumor burden $>2 \mathrm{~cm}$ ); and completeness of chemotherapy is categorized into completed and not completed. Table III contains the values of c-met overexpression. FIGO, International Federation of Gynecology and Obstetrics.

presented in absolute and relative numbers or as the median and quartiles. Comparison between clinicopathological factors and the overexpression of c-met was calculated using the $\chi^{2}$ test. The Cox proportional hazard regression model was used to evaluate the effect of investigative variables on progression-free survival (PFS) and disease-specific survival (DSS). PFS was defined as the time-frame between the date of the first histological proof of $\mathrm{OC}$ and the date of the first progression. DSS was defined as the time-frame between the date of the first histological proof of OC and the date of death due to OC. Univariate Cox-regression analysis was performed for every single variable. Variables with a P-value of $<0.05$ entered the multivariate Cox-regression analysis with a variable selection via backward elimination. All associations were reported as hazard ratios (HRs) with $95 \%$ confidence interval (CIs) and P-values. Kaplan-Meier estimations were performed to describe survival rates. As this was an investigative study, no adjustments for multiple testing were made. The statistical tests were performed for illustrative purposes only. P-values were awarded for descriptive reasons only and should be interpreted with caution and in connection with effect estimates.

\section{Results}

A total of 146 patients were screened. Of these, 8 and 29 patients were excluded due to missing tissue samples and incomplete follow-up information, respectively. A further 3 patients suffered from an ovarian borderline tumor. Therefore, 106 patients were enrolled in the study. The median follow-up time was 28.2 months. In this time, 69 (65.1\%) cases 
Table II. Patients' characteristics with c-met positive ovarian cancer.

\begin{tabular}{|c|c|c|c|c|c|c|c|c|}
\hline $\begin{array}{l}\text { Case } \\
\text { no. }\end{array}$ & $\begin{array}{c}\text { Expression } \\
\text { of c-met }\end{array}$ & $\begin{array}{l}\text { Age, } \\
\text { years }\end{array}$ & Histotype & Grading & Stage & $\begin{array}{l}\text { Post-operative } \\
\text { tumor } \\
\text { burden, } \mathrm{cm}\end{array}$ & $\begin{array}{c}\text { Completeness } \\
\text { of } \\
\text { chemotherapy }\end{array}$ & $\begin{array}{c}\text { Follow-up + time } \\
\text { after diagnosis, } \\
\text { years }\end{array}$ \\
\hline 1 & $2+$ & 47 & HGS & - & I & 0 & Yes & ALI, +6.9 \\
\hline 2 & $2+$ & 72 & HGS & - & III & $<2$ & Yes & $\mathrm{DOD},+1.2$ \\
\hline 3 & $2+$ & 75 & HGS & - & IV & $<2$ & No & $\mathrm{DOD},+2.4$ \\
\hline 4 & $2+$ & 63 & HGS & - & III & $<2$ & Yes & $\mathrm{AWD},+3.5$ \\
\hline 5 & $2+$ & 66 & HGS & - & III & $<2$ & Yes & $\mathrm{DOD},+8.5$ \\
\hline 6 & $2+$ & 55 & LGS & - & I & 0 & Yes & ALI, +1.4 \\
\hline 7 & $2+$ & 54 & LGS & - & III & $>2$ & Yes & $\mathrm{DOD},+5.0$ \\
\hline 8 & $2+$ & 65 & LGS & - & III & $>2$ & N/A & $\mathrm{DOD},+4.1$ \\
\hline 9 & $2+$ & 68 & LGS & - & III & 0 & Yes & $\mathrm{DOD},+0.4$ \\
\hline 10 & $2+$ & 77 & Mucinous & 1 & III & 0 & Yes & AWD,+1.4 \\
\hline 11 & $2+$ & 69 & Mucinous & 2 & III & 0 & Yes & $\mathrm{DOD},+7.6$ \\
\hline 12 & $2+$ & 48 & Mucinous & 2 & IV & $<2$ & Yes & ALI,+0.5 \\
\hline 13 & $2+$ & 36 & Mucinous & 2 & I & 0 & Yes & $\mathrm{ALI},+4.1$ \\
\hline 14 & $2+$ & 73 & Mucinous & 2 & I & 0 & Yes & $\mathrm{DOD},+1.2$ \\
\hline 15 & $2+$ & 24 & Mucinous & 2 & III & 0 & Yes & $\mathrm{AWD},+1.0$ \\
\hline 16 & $2+$ & 40 & Mucinous & 3 & III & $<2$ & Yes & $\mathrm{DOD},+0.5$ \\
\hline 17 & $2+$ & 65 & Endometrioid & 2 & IV & $<2$ & N/A & $\mathrm{DOD},+0.5$ \\
\hline 18 & $2+$ & 40 & Endometrioid & 2 & I & 0 & Yes & $\mathrm{ALI},+4.0$ \\
\hline 19 & $2+$ & 48 & Clear cell & 3 & III & 0 & Yes & $\mathrm{ALI},+4.0$ \\
\hline 20 & $2+$ & 71 & Mixed & 3 & III & $>2$ & Yes & $\mathrm{DOD},+4.0$ \\
\hline 21 & $3+$ & 52 & LGS & - & I & 0 & Yes & $\mathrm{DOD},+4.9$ \\
\hline 22 & $3+$ & 57 & Mucinous & 2 & I & 0 & Yes & ALI, +5.7 \\
\hline
\end{tabular}

HGS, high-grade serous; LGS, low-grade serous; ALI, alive; DOD, died of disease; N/A, not available.
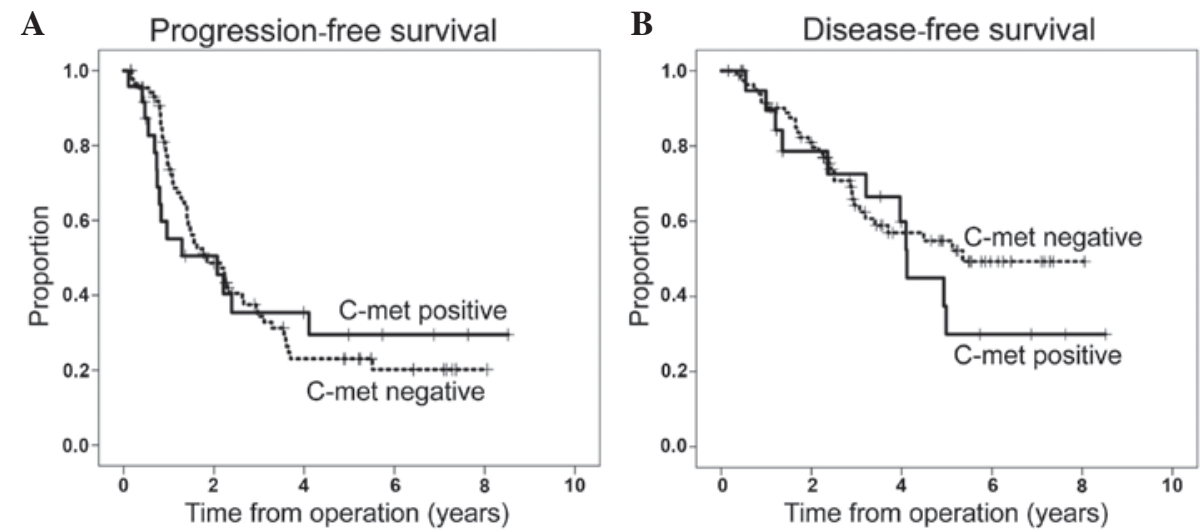

Figure 3. Kaplan-Meier plots of c-met with regard to (A) progression-free survival and (B) disease-specific survival for the entire cohort. The dashed lines represent the c-met-negative patients, whereas the continued lines represent the c-met-positive patients.

of recurrence and 44 (41.5\%) mortalities due to OC occurred. A total of 58 (54.7\%) patients exhibited high-grade serous OC, and 48 (45.3\%) patients exhibited type I OC. The characteristics of the patients are presented in Table I.

Overexpression of c-met was observed in 22 (20.8\%) cases. The characteristics of the patients with overexpression of c-met are presented in Table II. It became evident that 8 out of $14(57.1 \%)$ cases with mucinous OC and 5 out of $24(20.8 \%)$ cases with low-grade serous OC showed overexpression of c-met. In total, 17 out of 48 (35.4\%) cases displayed overexpression of c-met among the patients with type I OC, whereas only 5 out of $58(8.6 \%)$ of the cases with type II OC displayed c-met overexpression $(\mathrm{P}=0.001)$ (Table III and Fig. 2). Overexpression of c-met was not associated with any other clinicopathological features (Table III and Fig. 2). 
Table III. Clinicopathological factors with regard to the overexpression of c-met.

\begin{tabular}{|c|c|c|c|}
\hline Clinicopathological factor & Negative, $\mathrm{n}$ & Positive, n (\%) & P-value \\
\hline $\operatorname{Age}^{\mathrm{b}}$ & & & 0.406 \\
\hline$<59.08$ & 41 & $12(22.6)$ & \\
\hline$>59.08$ & 43 & $10(18.9)$ & \\
\hline Tumor stage (FIGO) & & & 0.344 \\
\hline $\mathrm{I}$ & 15 & $7(31.8)$ & \\
\hline II & 6 & $0(0.0)$ & \\
\hline III & 51 & $12(19.0)$ & \\
\hline IV & 12 & $3(20.0)$ & \\
\hline Histological grade $(n=24)^{c}$ & & & 0.122 \\
\hline 1 & 2 & $1(33.3)$ & \\
\hline 2 & 3 & $8(72.7)$ & \\
\hline 3 & 7 & $3(30.0)$ & \\
\hline Histological type ${ }^{b}$ & & & $0.001^{\mathrm{d}}$ \\
\hline Type I & 31 & $17(35.4)$ & \\
\hline Type II & 53 & $5(8.6)$ & \\
\hline Post-operative residual tumor burden & & & 0.834 \\
\hline R0 & 50 & $12(19.4)$ & \\
\hline $\mathrm{R} 1$ & 26 & $7(21.2)$ & \\
\hline $\mathrm{R} 2$ & 8 & $3(27.3)$ & \\
\hline Completeness of chemotherapy $(n=105)^{b}$ & & & 0.186 \\
\hline Yes & 12 & $1(7.7)$ & \\
\hline No & 63 & $19(23.2)$ & \\
\hline
\end{tabular}

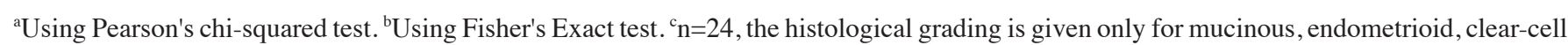
and mixed cases in accordance to the current FIGO classification ${ }^{\mathrm{d}} \mathrm{P}<0.05$. FIGO, International Federation of Gynecology and Obstetrics; R0, no tumor burden; R1, residual disease $<2 \mathrm{~cm}$; R2, residual disease $>2 \mathrm{~cm}$.

According tounivariateCox-regressionanalysis,c-met was not associated with PFS or DSS $(\mathrm{P}=0.835$ and 0.414 , respectively) (Table IV). According to multivariate Cox-regression analysis, only tumor stage and post-operative tumor burden retained significance in terms of $\mathrm{PFS}(\mathrm{P}=0.001$ and $\mathrm{P}<0.001$, respectively), with $\mathrm{HRs}$ of 1.608 (95\% CI, 1.210-2.138) and 1.915 (95\% CI, 1.345-2.728), respectively. In terms of DSS, post-operative tumor burden and completeness of chemotherapy retained their significance $(\mathrm{P}=0.003$ and $\mathrm{P}=0.012$, respectively), with HRs of 2.077 (95\% CI, 1.284-3.360) and 0.321 (95\% CI, 0.133-0.776), respectively (Table IV). Accordingly, Kaplan-Meier plots failed to demonstrate an effect of c-met, with no significant difference between c-met-positive and c-met-negative cases, respectively, in terms of 5-year PFS rate $(24.2$ vs. $32.5 \%, \mathrm{P}=0.938)$ and 5 -year DSS rate (54.8 vs. $29.9 \%, \mathrm{P}=0.412$ ) (Fig. $3 \mathrm{~A}$ and $\mathrm{B}$ ). Furthermore, c-met expression did not alter the 5-year PFS and DSS rates among any subgroups, including the high-grade serous OC or non-high-grade serous OC groups (data not shown).

\section{Discussion}

To the best of our knowledge, the present study reports for the first time that the overexpression of c-met is significantly associated with type I OC in comparison with type II OC.
The study results support the hypothesis that the overexpression of c-met is involved in the carcinogenesis of type I OC. This may be due to the fact that c-met is involved in cross-talks with several pathways, such as RAS-MAPK and PI3K-AKT $(7,9)$, known to impact the development of type I OC subtypes (3-5).

Results of studies by Yamamoto et al (13) and Sawada et al (14) are in agreement with the present study observations. To the best of our knowledge, Yamamoto et al (13) reported the largest study of c-met overexpression in non-serous OC: In a consecutive cohort of 201 OC patients, a large subset of 90 patients exhibited clear-cell OC. Not unexpectedly, the reported c-met protein levels were comparable with the present study results: C-met overexpression was detected in $22 \%$ of clear-cell OC but not in any non-clear cell $\mathrm{OC}$, and copy number alterations were detectable in $24 \%$ of the clear cell OC and in $3 \%$ of the non-clear cell OC, respectively (13). In 2007, Sawada et al (14) published the results of c-met overexpression in a cohort of $138 \mathrm{OC}$ patients. Within this cohort, $82.6 \%$ patients exhibited serous OC and $69.6 \%$ exhibited poorly-differentiated OC. Comparable with the present results, the study reported c-met overexpression in only $11 \%$ of cases $(15 / 138)$. Notably, a higher proportion of patients with mucinous and clear cell OC showed overexpression of $c$-met $(3 / 10$ and $1 / 3$, 
Table IV. Univariate and multivariate Cox-regression analysis for progression-free and disease-specific survival.

A, Progression-free survival

\begin{tabular}{|c|c|c|c|c|}
\hline \multirow[b]{2}{*}{ Parameter } & \multicolumn{2}{|c|}{ Univariate } & \multicolumn{2}{|c|}{ Multivariate } \\
\hline & HR $(95 \% \mathrm{CI})$ & $\mathrm{P}$-value & HR $(95 \%$ CI $)$ & P-value \\
\hline Age, years & $0.730(0.455-1.172)$ & 0.192 & - & \\
\hline Tumor stage (FIGO) & $1.825(1.406-2.368)$ & $<0.001^{\mathrm{a}}$ & $1.608(1.210-2.138)$ & $0.001^{\mathrm{a}}$ \\
\hline Histological grade & $1.518(0.482-4.782)$ & 0.476 & - & \\
\hline Histological type & $0.692(0.423-1.132)$ & 0.142 & - & \\
\hline Post-operative residual tumor burden & $2.420(1.744-3.358)$ & $<0.001^{\mathrm{a}}$ & $1.915(1.345-2.728)$ & $<0.001^{\mathrm{a}}$ \\
\hline Completeness of chemotherapy & $0.547(0.278-1.077)$ & 0.081 & - & \\
\hline c-met & $0.938(0.511-1.719)$ & 0.835 & - & \\
\hline
\end{tabular}

B, Disease-specific survival

\begin{tabular}{|c|c|c|c|c|}
\hline \multirow[b]{2}{*}{ Parameter } & \multicolumn{2}{|c|}{ Univariate } & \multicolumn{2}{|c|}{ Multivariate } \\
\hline & $\mathrm{HR}(95 \% \mathrm{CI})$ & P-value & HR $(95 \% \mathrm{CI})$ & P-value \\
\hline Age, years & $0.569(0.311-1.040)$ & 0.067 & - & \\
\hline Tumor stage (FIGO) & $1.986(1.387-2.843)$ & $<0.001^{\mathrm{a}}$ & $1.396(0.898-2.170)$ & 0.138 \\
\hline Histological grade & $1.251(0.329-4.764)$ & 0.742 & - & \\
\hline Histological type & $1.002(0.551-1.821)$ & 0.996 & - & \\
\hline Post-operative residual tumor burden & $2.135(1.440-3.168)$ & $<0.001^{\mathrm{a}}$ & $2.077(1.284-3.360)$ & $0.003^{\mathrm{a}}$ \\
\hline Completeness of chemotherapy & $0.312(0.141-0.688)$ & $0.004^{\mathrm{a}}$ & $0.321(0.133-0.776)$ & $0.012^{\mathrm{a}}$ \\
\hline c-met & $1.330(0671-2.637)$ & 0.414 & - & \\
\hline
\end{tabular}

${ }^{a} \mathrm{P}<0.05$. FIGO, International Federation of Gynecology and Obstetrics; HR, hazard ratio; CI, confidence interval.

respectively) (14). However, potential limitations of these two studies result from the fact that no further differentiation between high-grade and low-grade serous OC was performed. Furthermore, the latter study had only a small proportion of patients with non-serous OC. By contrast, Goode et al (15) reported a notably high rate of $97.8 \%$ for c-met overexpression in a cohort of $>320$ consecutive OC patients (moderate staining in $38.9 \%$ and strong staining in $58.9 \%$ of the entire cohort). However, no further differentiation into the various histological subtypes of OC was provided. In summary, the available literature on the pattern of expression of c-met in OC is partially contradictory (13-15).

The present study performed Cox-regression analyses and Kaplan-Meier estimations to investigate the potential prognostic impact of c-met. According to the data, overexpression of c-met failed to exhibit any prognostic impact in the entire cohort, as well as in the subsets of type I or type II OC, respectively. In line with this analysis, Goode et al (15) were not able to detect an association of genotype, protein expression and mortality within a consecutive cohort of $>320$ patients. Conversely, Yamamoto et al (13) reported an independent negative prognostic effect of c-met, as evaluated by immunohistochemistry, on overall survival in the subset of clear cell carcinoma $(\mathrm{P}=0.018)$. Supporting the present results, the study failed to demonstrate any further prognostic impact of c-met in the entire cohort or in the subset of non-clear cell $\mathrm{OC}$ in terms of PFS (13). Sawada et al (14) showed an independent impaired effect of c-met overexpression in terms of overall survival $(\mathrm{P}=0.005)$, but not in terms of PFS. Within this cohort, a notably high rate of $82.6 \%$ of serous OC cases and a high rate of $69.6 \%$ of histological poorly-differentiated OC patients were included (14). Arguably, potential limitations of these two studies arise from the fact that no further differentiation between high-grade and low-grade serous OC was performed and that the number of patients presenting with non-serous OC was notably low or was not reported.

Overall, in the present study, the prognostic effect of the post-operative tumor burden, the tumor stage and the completeness of chemotherapy, as well as the low rate of c-met positive cases, are in line with the published literature. However, limitations result from the retrospective design of the study. In order to reduce a bias resulting from incomplete follow-up, patients with incomplete follow-up were excluded from the study.

In conclusion, to the best of our knowledge, the present study shows for the first time that c-met was predominantly overexpressed in type I OC, supporting the hypothesis that c-met has a role in the development of type I OC. However, c-met overexpression was not associated with prognosis in this disease. 


\section{Acknowledgements}

The abstract was presented at the Annual Meeting of Deutsche Krebsgesellschaft Feb 2016 in Berlin and published as abstract no. 0194 in Oncol Res Treat (Suppl 1): 2016. This study was supported in part by a grant from the University of Heidelberg (Heidelberg, Germany; grant no. 20080715). The authors would like to thank Ms. Susanne Gebhard and Ms. Martina Seehase (Department of Gynecology and Obstetrics, University Medical Center Mainz, Mainz, Germany) for providing technical assistance and support in data management.

\section{References}

1. Siegel R, Ma J, Zou Z and Jemal A: Cancer Statistics, 2014. CA Cancer J Clin 64: 9-29, 2014.

2. Piek JM, Kenemans P and Verheijen RH: Intraperitoneal serous adenocarcinoma: A critical appraisal of three hypotheses on its cause. Am J Obstet Gynecol 191: 718-732, 2004.

3. Levanon K, Crum C and Drapkin R: New insights into the pathogenesis of serous ovarian cancer and its clinical impact. J Clin Oncol 26: 5284-5293, 2008.

4. Kurman RJ: Origin and molecular pathogenesis of ovarian high-grade serous carcinoma. Ann Oncol 24 (Suppl 10): x16-x21, 2013.

5. Landen CN Jr, Birrer MJ and Sood AK: Early events in the pathogenesis of epithelial ovarian cancer. J Clin Oncol 26 : 995-1005, 2008.

6. Ahmed AA, Etemadmoghadam D, Temple J, Lynch AG, Riad M, Sharma R, Stewart C, Fereday S, Caldas C, Defazio A, et al: Driver mutations in TP53 are ubiquitous in high grade serous carcinoma of the ovary. J Pathol 221: 49-56, 2010.

7. Gherardi E, Birchmeier W, Birchmeier C and Vande Woude G: Targeting MET in cancer: Rationale and progress. Nat Rev Cancer 12: 89-103, 2012.

8. Ma PC, Tretiakova MS, Mackinnon AC, Ramnath N, Johnson C, Dietrich S, Seiwert T, Christensen JG, Jagadeeswaran R, Krausz T, et al: Expression and mutational analysis of MET in human solid cancers. Genes Chromosomes Cancer 47: 1025-1037, 2008.
9. Birchmeier C, Birchmeier W, Gherardi E and Vande Woude GF: Met, metastasis, motility and more. Nat Rev Mol Cell Biol 4: 915-925, 2003

10. Lai AZ, Abella JV and Park M: Crosstalk in Met receptor oncogenesis. Trends Cell Biol 19: 542-551, 2009.

11. Trusolino L, Bertotti A and Comoglio PM: MET signalling: Principles and functions in development, organ regeneration and cancer. Nat Rev Mol Cell Biol 11: 834-848, 2010.

12. Buckanovich R, Berger R, Sella A, Sikic B, Shen X, Ramies D, Smith DC and Vergote IB: Activity of cabozantinib (XL184) in advanced ovarian cancer patients (pts): Results from a phase II randomized discontinuation trial (RDT). J Clin Oncol 29 (suppl): abstract 5008, 2011

13. Yamamoto S, Tsuda H, Miyai K, Takano M, Tamai S and Matsubara O: Gene amplification and protein overexpression of MET are common events in ovarian clear-cell adenocarcinoma: Their roles in tumor progression and prognostication of the patient. Mod Pathol 24: 1146-1155, 2011.

14. Sawada K, Radjabi AR, Shinomiya N, Kistner E, Kenny H, Becker AR, Turkyilmaz MA, Salgia R, Yamada SD, Vande Woude GF, et al: c-Met overexpression is a prognostic factor in ovarian cancer and an effective target for inhibition of peritoneal dissemination and invasion. Cancer Res 67: 1670-1679, 2007.

15. Goode EL, Chenevix-Trench G, Hartmann LC, Fridley BL, Kalli KR, Vierkant RA, Larson MC, White KL, Keeney GL, Oberg TN, et al: Assessment of hepatocyte growth factor in ovarian cancer mortality. Cancer Epidemiol Biomarkers Prev 20: 1638-1648, 2011.

16. Battista MJ, Mantai N, Sicking I, Cotarelo C, Weyer V, Lebrecht A, Solbach C and Schmidt M: Ki-67 as an independent prognostic factor in an unselected cohort of patients with ovarian cancer: Results of an explorative, retrospective study. Oncol Rep 31: 2213-2219, 2014.

17. Battista MJ, Cotarelo C, Jakobi S, Steetskamp J, Makris G, Sicking I, Weyer V and Schmidt M: Overexpression of epithelial cell adhesion molecule protein is associated with favorable prognosis in an unselected cohort of ovarian cancer patients. J Cancer Res Clin Oncol 140: 1097-1102, 2014.

18. Malpica A, Deavers MT, Lu K, Bodurka DC, Atkinson EN, Gershenson DM and Silva EG: Grading ovarian serous carcinoma using a two-tier system. Am J Surg Pathol 28: 496-504, 2004. 